\title{
Propuesta de modelo documental de gestión de mejora con enfoque sistémico
}

\section{Proposal for a model of improvement management with systemic approach}

\author{
LAGUNA-AGUILAR, Fabiola María del Carmen*†, LARA-GÓMEZ, Graciela y MONTAÑO- \\ ARANGO, Oscar \\ Cuerpo Académico Optimización de Procesos Productivos de la Universidad Tecnológica Tula-Tepeji. Avenida \\ Universidad Tecnológica, El Carmen, $42830 \mathrm{Hgo.}$ \\ Cuerpo Académico Organización y Desarrollo de la Universidad Autónoma de Querétaro. Centro Universitario, Cerro de \\ las Campanas s/n C.P. 76010, Cto Universitario, Centro Universitario, 76010 Santiago de Querétaro, Qro. \\ Cuerpo Académico de Ingeniería de Sistemas Organizacionales de la Universidad Autónoma del Estado de Hidalgo. \\ Pachuca - Actopan Km. 4.5, Campo de Tiro, 42039 Pachuca de Soto, Hgo.
}

ID $1^{\text {er }}$ Autor: Fabiola María del Carmen, Laguna-Aguilar / ORC ID: 0000-0002-7210-0081, Researcher ID Thomson: X6195-2018, CVU CONACYT ID: 307845

ID $1^{\text {er }}$ Coautor: Graciela, Lara- Gómez / ORC ID: 0000-0001-9984-7372, CVU CONACYT ID: 99837

ID $3^{\text {er }}$ Coautor: Oscar, Montaño-Arango / CVU CONACYT ID: 35755

DOI: $10.35429 /$ JOTE.2019.9.3.17.26

Recibido 15 de Julio, 2019; Aceptado 25 de Septiembre, 2019

\section{Resumen}

La globalización exige a las organizaciones reaccionar rápida y constantemente para sobrevivir, existe la necesidad de optimizar productos y procesos para ser tanto productivas como competitivas; proporcionar valor agregado y mantenerse en el mercado. Este artículo se genera a partir de una investigación documental sobre modelos de mejora continua, con el propósito de servir como referencia para proponer un modelo documental de gestión de mejora con enfoque sistémico, factible de aplicar en una organización de Educación Superior pública que permita incrementar indicadores de productividad organizacional; lograr objetivos y metas planteadas al integrar, trabajo colaborativo, comunicación efectiva entre todas las partes interesadas (clientes, proveedores, sociedad, personal, competencia, usuarios etc.). Seguimiento mediante uso de tecnologías de la información, brindar reconocimiento al logro del personal; posibilidad de gestionar la mejora desde la parte operativa y evaluar el nivel de madurez de la organización para la mejora.

Modelos de madurez, Mejora continua, Productividad, Organizaciónal, Sistémico

\begin{abstract}
The current globalized world requires organizations to apply strategies that allow them to live competitively, which makes it necessary to continuously improve products and processes in order to be productive; providing added value and thus stay in the market. The article is generated from a documentary research on models of continuous improvement triggers of productivity, with the purpose of serving as a reference to propose a document model of improvement management with a systemic approach feasible to apply in a public higher education organization that allow to increase the established organizational productivity indicators; reflected in the achievement of objectives and goals set to integrate, collaborative work, effective communication among all stakeholders (customers, suppliers, society, staff, competition, users, etc.). Follow-up, through the use of information technology, providing recognition to staff achievement; possibility of managing the improvement from the operative part. Evaluate the level of maturity for improvement.
\end{abstract}

Models of maturity, Continuous improvement, Productivity, Organizational, Systemic

Citación: LAGUNA-AGUILAR, Fabiola María del Carmen, LARA-GÓMEZ, Graciela y MONTAÑO- ARANGO, Oscar. Propuesta de modelo documental de gestión de mejora con enfoque sistémico. Revista de Educación Técnica. 2019. 3-9: 1724.

\footnotetext{
*Correspondencia al Autor (Correo Electrónico: fabiolamaria.laguna@uttt.edu.mx)

$\uparrow$ Investigador contribuyendo como primer autor.
} 


\section{Introducción}

En el mundo globalizado actual las organizaciones que sobreviven son aquellas que son competitivas al ser productivas, porque ofrecen valor agregado a sus clientes y a las partes interesadas, para generar lealtad que permita la permanencia en el mercado; lo que implica la aplicación de círculos virtuosos de mejora continua. La mejora continua es importante por la velocidad actual a la que ocurren los avances tecnológicos lo que implica una actualización y adaptación de los procesos, así como de los productos o servicios que se ofrecen para no quedar obsoletos sin capacidad de responder a las necesidades de los usuarios, dando como consecuencia baja productividad, dejando de ser competitivos por no ofrecer ventajas competitivas, es decir valor agregado; con productos diferenciados que los mantengan en la preferencia de sus consumidores. La gestión y mejora de procesos formal debe estar fundamentada en supuestos teóricos con enfoque de procesos e integración de sistemas, que permita evaluar oportunidades de mejora, con indicadores alineados a la planeación estratégica de una organización. La investigación se encuentra fundamentada en un trabajo previo de una propuesta de un modelo de sana convivencia y gestión humana con enfoque sistémico como detonador de la productividad organizacional.

\section{Justificación}

La Globalización ha originado una evolución más rápida en los ámbitos político económico, social, tecnológico, cultural, ecológico y educativo, por la velocidad a la que viaja actualmente la información y con el uso de las tecnologías de la información, permitiendo actualmente avances tecnológicos que se dan en menos de un lustro; esto obliga a las organizaciones públicas y privadas a estar realizando cambios constantes para satisfacer las necesidades de los clientes, que implica tomar acciones que le permitan adaptarse rápidamente realizando ajustes y mejoras en sus productos, así como en sus procesos para ser competitivos, mantenerse en el mercado por preferencia de sus consumidores con productos o servicios que proporcionen alto valor agregado o diferenciación, que genera la lealtad de los clientes o usuarios para lograr su supervivencia sin obsolescencia.
Todo ello hace necesario aplicar modelos de mejora continua que se adapten a las características de la organización, en este caso particular a instituciones de Educación Superior ocupadas por mantenerse competitivas y a la vanguardia después de identificar y reconocer sus áreas de oportunidad.

\section{Problema}

Una organización de Educación Superior pública que forma profesionistas con competencias para insertarse en empresas tanto nacionales como internacionales con intercambios de alumnos así como de personal dentro y fuera de su nación, reconoce como área de oportunidad la necesidad de contar con trabajo colaborativo de alto impacto que se refleje en indicadores y logros institucionales, Así como la falta de regulación de incentivos para motivar la participación en la realización de proyectos; dentro del (Programa Institucional de Desarrollo) PIDE 2017-2022.

\section{Hipótesis}

Un modelo de mejora continua que alinee los objetivos del personal a los objetivos institucionales podrá impactar en la productividad organizacional.

\section{Objetivos}

\section{Objetivo General}

Desarrollar una investigación documental del estado del arte acerca de los modelos de mejora continua para fundamentar un nuevo modelo de mejora continua que alinee los objetivos del personal a los objetivos institucionales con la finalidad de fortalecer la productividad organizacional.

\section{Objetivos específicos}

- Estudiar la evolución de los modelos de mejora continua para tener un marco de referencia.

- Analizar el contexto de la aplicación de los modelos de mejora continua en las organizaciones para fundamentar la evaluación de la cultura organizacional. 
- Analizar el impacto que ha tenido la aplicación de los modelos de mejora continua en la productividad.

- Identificar las diferencias entre propuestas de modelos para integrar una propuesta integral acorde a las necesidades de la organización en cuestión.

- Diseñar un esquema de modelo de mejora que relacione los objetivos de la organización con los objetivos de los miembros de la misma, que pueda ser aplicado a cualquier organización para elevar su productividad.

\section{Materiales y métodos}

De acuerdo con Navarro (2017) en este trabajo se realizó una revisión bibliográfica y documental acerca de los modelos de mejora continua y sus efectos en la productividad de una organización con la metodología analítica y el procedimiento inductivo-deductivo de búsqueda de las tendencias actuales en la temática abordada; que permita una perspectiva teórica a partir del análisis de las siguientes fuentes:

La Globalización obliga buscar la mejora continua para mantener competitividad con productividad para proporcionar valor agregado, mantenerse en el mercado, crecer y desarrollarse. (Fontalvo, J.T., 2006).

De acuerdo con Fontalvo J.T. (2006) el concepto de calidad ha evolucionado a lo largo de los dos últimos siglos y lo define como "el conjunto de características inherentes a un producto o servicio que están en capacidad de garantizar el cumplimiento de las necesidades y expectativas de los clientes, de los miembros de la empresa y de la sociedad" (Fontalvo, J.T., 2006, p. 38). Que se logra al proporcionarles un mayor nivel de satisfacción.

Desde el enfoque de valor agregado para el cliente o usuario son el conjunto de características que generan la preferencia de los clientes sobre otros productos al cumplir plenamente con sus requisitos.

Para la organización internacional de normalización ISO la calidad es el "conjunto de características de un producto o servicio destinados a satisfacer la necesidades explicita e implícitas preestablecidas por cliente o usuario".
La International Standar Organization (ISO) (en su norma 8402), define la calidad como: "el conjunto de características de una entidad que le confieren la aptitud para satisfacer las necesidades establecidas $\mathrm{o}$ implícitas".

Fontalvo, J.T (2006). Describe cómo la estrategia de una organización se traduce en una ventaja competitiva al operar procesos interactivos que se retroalimentan con información para la mejora continua de la calidad que aportan valor agregado para el cliente y competitividad. Define a la competitividad como "capacidad de la empresa para producir productos o servicios mejores a los de la competencia con el menor costo posible por el uso racional de recursos". Con calidad, productividad y competitividad la empresa se mantiene en el mercado, crece y se desarrolla.

En el siglo pasado en los 50's se empezó a hablar de calidad principalmente en los Estados Unidos; para la industria de dicho país, control total de la calidad significaba definir bien las características del producto desde el diseño para evitar problemas en la fabricación y controlar los procesos con inspecciones y pruebas. Después de la segunda guerra mundial, Japón al perder la guerra quedó devastado por lo que fueron apoyados por ingenieros $\mathrm{y}$ estadistas estadounidenses como Deming y Juran para aprender prácticas de gestión que integraban el control estadístico de proceso; que eran utilizadas en empresas de los Estados Unidos en la búsqueda de la mejora continua; las cuales acogieron con disciplina para levantar a su país de la situación en la que se encontraba, estas prácticas fueron mejoradas y perfeccionadas integrando otras como los círculos de calidad y la filosofía "Just in Time". Después de 1980 surgió el concepto de calidad total.

PÉREZ-TORRES, Roxana, VARGAS-VILCHIS, Emmanuel, REYESDE LOS SANTOS, Iyeliz y VÁZQUEZ-SALAZAR, Roberto Salomón. Sistema de monitoreo y control de proyectos integradores en el ámbito académico. Revista de Educación Técnica. 2019 
Deming G.E, (1986). Propone el ciclo PDCA (Plan, Do, Check, Act) de planear, hacer, verificar y actuar en su libro "Calidad, Productividad y Competitividad: La salida de la crisis" como método para lograr la mejora continua el cual se difunde de forma masiva por lo que se hace popular como el circulo de Deming, sin embargo el autor original de este es Walter A. Shewart padre del control estadístico de la calidad que lo plantea a finales de la década de los 30's del siglo pasado en los laboratorios Dell Telephone de la Western electric Company donde trabajaba conjuntamente con Deming, siendo este último su discípulo; publicando el libro "Métodos estadísticos desde el punto de vista de control de calidad" en 1939.

Deming G.E. (1989) Menciona dentro de sus 14 puntos en el quinto "Mejorar constantemente y siempre el sistema de producción y servicio, para mejorar la calidad y la productividad al reducir constantemente los costos".

En 1987 en Estados Unidos se genera el modelo de gestión de la excelencia Malcolm Baldrige para que las empresas lograran ser más competitivas y hacer frente a las japonesas con un gran avance en lo que llamaban Calidad Total. Aunque este modelo de gestión de la excelencia nació en el sector productivo, se utilizó en el sector de servicios como la salud y la educación. (López, 2001).

El modelo llegó a Europa cerca de 1990 con el nombre de Modelo EFQM (European Fundation for Quality Management) Fundación europea de la gestión de la calidad. En 1999 surge el modelo iberoamericano FUNDIBEQ (Fundación Iberoamericana par la gestión de la calidad) con sede en España; de acuerdo FUNDIBEQ (2015) integra la experiencia de otros países en implantar modelos y sistemas de excelencia para mejorar su competitividad y consolidarla de forma internacional. Este modelo es adoptado y adaptado según sus características en países como Argentina, Paraguay, Brasil, Uruguay, Colombia, Chile y México. (Fundación Iberoamericana para la Gestión de la Calidad, 2015).
Los modelos más importantes que permiten la implementación de la Gestión de la Calidad Total (GCT) en una organización son modelos reconocidos y utilizados, como referencia porque establece criterios de evaluación para otorgar distintos premios internacionales a la calidad con el propósito de otorgar reconocimiento a las empresas ejemplares en Gestión de la Calidad. (Bohoris, 1994). El Modelo Deming Prize japonés fue el primero que nació en 1951. El modelo Malcolm Baldrige en 1987, fundamento del Malcolm Baldrige Quality National Award (Estados Unidos), el modelo de Excelencia de la European Foundation for Quality Management (EFQM), estructura de base para la presentación al European Quality Award de creado en 1999, el modelo Iberoamericano a la Excelencia en la Gestión, auspiciado por la Fundación Iberoamericana para la Gestión de la Calidad (FUNFIBEQ) en el cual se basa el Premio Iberoamericano de la Calidad. (López, 2001). En México existe el Modelo Nacional de Competitividad para el premio Nacional de Calidad y los Premios estatales de calidad.

Los modelos se aplican a instituciones públicas y privadas sin embargo para las organizaciones públicas tienen diferencias muy particulares para proporcionar calidad y excelencia en los servicios públicos con el fomento de una cultura de calidad total, por ello se han desarrollado modelos como INTRAGOB (2000-2006) con criterios con fundamento en el Premio Nacional de Calidad dirigidos a la Administración Pública Federal. (Sánchez, A.; Rincón, M. \& González, F., 2005). También existen modelos de GCT a nivel nacional, como el Premio Príncipe Felipe a la Excelencia Empresarial y al Sello de Excelencia Europea que concede el Club de Excelencia en Gestión. Estos modelos facilitan la implantación de sistemas de GCT a través de la descripción de criterios, porque presentan una sistematización global para ello, y proporcionar un método para medir el progreso de la organización que sirven de autoevaluación al identificar fortalezas y debilidades que son una base para definir estrategias y acciones de mejora, innovación y aprendizaje al comparar las actividades y resultados de una organización con los criterios del modelo de excelencia; permiten desarrollar estrategias para la calidad, para elaborar el plan de GCT. (Bohoris, 1995) 
Existen varios conceptos relacionados a la mejora contínua. En sus inicios estaban relacionados a la calidad; en su factor número doce " tener un proceso y herramientas para el mejoramiento permanente" (Ishikawa, 1991) y (Juran, 1990) en su décimo principio "Mantener el impulso al hacer que la mejoría anual sea parte de los sistemas y procesos regulares de la compañía" siendo uno de los tres procesos para administrar la calidad " la mejora de la calidad" con la infraestructura necesaria, identificando los proyectos de mejora, proporcionando recursos, motivación y formación que permitan hacer un diagnóstico de las causas, remediarlas y establecer controles para mantener beneficios; reconociendo la necesidad del compromiso del personal. La ISO 9000:2000 define la mejora continua como una actividad recurrente para aumentar la capacidad de cumplir requisitos que es fundamental para implantar sistemas gestión normalizados, con un aprendizaje continuo de la organización que nunca termina definitivamente.

La mejora continua implica realizar cambios organizados de forma proactiva y no reactiva para tener un mayor desempeño identificando áreas de oportunidad sin necesidad de detonarse a partir de una problemática por incumplimiento o presencia de defectos que implican rechazos, reclamos y costos, siendo el motor impulsor eliminar actividades o circunstancias que no agregan valor al producto convirtiéndose en un ciclo virtuoso.

En los 90's del siglo pasado se vuelve popular el termino reingeniería de negocios (Babé, 2002), que consiste en volver a diseñar procesos para optimizar los recursos y mejorar la satisfacción del cliente así como las partes interesadas; implica una metodología con cinco fases, donde la primera requiere en hacer un diagnóstico de las áreas susceptibles a mejorar, en la segunda se definen el equipo de trabajo, los objetivos, las estrategias el plan de trabajo, la tercera fase es el desarrollo que implica entrenamiento con nuevos procedimientos de operación, en la cuarta fase se da la implantación con la comunicación de los nuevos procedimientos y en la fase final se crean equipos de mejora continua de los procesos y sistema.
Desde el enfoque japonés la reingeniería significa innovación o cambio radical (Ishikawa, 1991). La reingeniería es una conceptualización de la innovación japonesa. Para (Babé, 2002) es trabajo duro, que se presenta en forma planeada y programada más que con arreglos precipitados.

De acuerdo con Champy (1995) existen cuatro aspectos que se deben considerar para tener éxito al hacer reingeniería que son: cuestionar el propósito y razón de ser de lo que se hace en la organización, el cambio cultural que necesita la organización y definición de personal que se requiere, crear nuevos estándares y procedimientos, y practicar los nuevos procedimientos.

Al aplicar un cambio radical, el rendimiento, disminuye por la curva de aprendizaje, por lo que un programa de reingeniería debe estar precedido por una serie de mejoras continuas (Cantú, 2001). La mejora es un proceso de toma de decisiones que se realiza en función de las necesidades de cada organización.

Existen diversas herramientas para la gestión y mejora de los procesos que se clasifican en metodologías, modelos y procedimientos; La diversidad de herramientas para la gestión y mejora existe porque cada organización tiene características distintas, a pesar de que sus productos sean similares. Sin embargo tienen en común estar fundamentadas el ciclo de mejora continua de Deming de Planear, Ejecutar, Verificar y Mejorar, de manera cíclica (Ishikawa, 1991).

La gestión de mejora implica asegurar el análisis, la verificación y eliminación de probables fallas; con el uso de controles para supervisar y administrar el proceso mejorado; requiere permanente capacitación y la actualización de la documentación que se requiere cada ciclo de mejora; evita que vuelvan a aparecer las causas que de los problemas, al estandarizar los procesos mejorados.

Medina León y otros (2014), muestran la evolución en los procedimientos de mejora de procesos desde finales del siglo pasado hasta la fecha. Al inicio eran generales sin medidas concretas para la implementación de acciones de mejora. 
Con el paso del tiempo y con nuevos conceptos en la gestión empresarial comenzaron a ser más específicos y orientadores, muchos de ellos se han adaptado para otros sectores. Sin embargo, tienen puntos en común como: el ciclo de mejora; la identificación de sus procesos y su interrelación; nombrar responsables de los procesos y las acciones de mejora; establecer indicadores para el control así como en el reconocimiento del personal.

El inicio de las acciones de mejora se pueden detonar a partir de la presencia de un conjunto de problemas que se priorizan con el análisis de las causas, o para atacar situaciones emergentes, o bien a partir del análisis de los indicadores empresariales.

Se requiere de la evaluación de las posibles acciones de mejora porque no todas se pueden aplicar al mismo tiempo, por razones de tiempo, financieras, disponibilidad de personal o relación con otros procesos o sistemas que afectarían la eficiencia.

En la aplicación de modelos de mejora se presenta resistencia al cambio, sobre todo cuando no existe comunicación y retroalimentación durante los ciclos de mejora. Por tanto, resulta imprescindible el diseño de modelos y procedimientos de mejora de la gestión de los procesos, ajustados a las nuevas necesidades de todas las partes interesadas y a los requerimientos propios de la entidad donde se implementen.

También en las normas internacionales (ISO, 2008) e (ISO, 2015) se fomentan la adopción de este enfoque cuando se implementa y mejora el Sistema de Gestión de Calidad, concentrándose en: 1. La comprensión y el cumplimiento de los requisitos de los clientes de cada proceso. 2. La necesidad de considerar y de planificar los procesos en términos que aporten valor (el cliente no debe pagar por algo que no le aporte valor). 3. El control, la medición y la obtención de resultados del desempeño y de la eficacia de los procesos. 4. La mejora continua de los procesos con base en mediciones objetivas. 5. Habilidad para concentrar los esfuerzos en los procesos claves. 6. La integración y alineación de los procesos, que mejor alcancen los resultados deseados. 7. Aumentar la capacidad de usar los mismos recursos.

\section{Resultados y discusión}

Se puede apreciar que existe coincidencia en los procedimientos o sistemas de gestión y mejora de procesos que hablan de etapas cíclicas con fundamento en el ciclo de mejora continúa propuesto por Shewar y difundido por Deming con las fases o etapas de planear, ejecutar, verificar y mejorar.

Los criterios de las mejores prácticas de gestión de mejora de los diferentes modelos investigados actualmente tienen un enfoque de procesos, donde existen elementos de entrada, que son salida de otro proceso relacionado; elementos de transformación o aplicación que influyen en los resultados como elementos de salida que a su vez se convierten en entradas de otros procesos relacionados dentro de una cadena de valor; por lo que deben ser claramente definidos y evaluados para identificar oportunidades de mejora, mediante el uso de indicadores o métricas para medirlos y monitorear su alineación con la administración estratégica de la empresa y estandarizar actividades en procedimientos documentados.

Las metodologías y modelos de mejora continua consideran al factor humano un elemento sustancial porque implica la modificación de sus actitudes, aptitudes y comportamiento para aprovechar mejor los recursos reduciendo costos e incrementando el valor de sus productos impactando en los indicadores de productividad, al lograr eficiencia y eficacia mejorando los resultados para todas las partes interesadas (clientes, inversionistas, personal, sociedad, proveedores, administradores).

La mejora contínua es una filosofía de trabajo reflejado en las acciones diarias con un comportamiento proactivo de propuestas y acciones que busquen el incremento de la satisfacción de las partes interesadas que se refleja en la velocidad de cambio con efectividad impactando en la productividad y competitividad convirtiéndose en un estilo de vida que además reconoce las aportaciones de las personas con beneficios que estimulan la participación en este círculo virtuoso. 
Los modelos de mejora continua se adaptan a cada organización aunque las fases en esencia sean las mismas propuestas por Sheward y Deming; las características de cada organización son diferentes, la investigación coincide que se requiere para la aplicación de las fases que la empresa esté bien organizada en su procesos productivos y administrativos, inversión para capacitación en el desarrollo de acciones de mejora, las herramientas estadísticas y las fases son una guía para mejorar dentro de las fases de planear, ejecutar, verificar y mejorar. La investigación documental permite retomar el modelo planteado en una investigación anterior LagunaAguilar, F. (2018) "Gestión Humana para Incremento de Productividad con enfoque sistémico". Adaptándolo Ver fig. 1 que relacione los objetivos de la organización con los intereses de su personal mediante 7 etapas. 1. Definir los objetivos, indicadores de productividad y recursos disponibles para las acciones de mejora y reconocimiento así como las políticas 2. Diagnosticar y solicitar propuestas para logro o mejora de indicadores con empleados. 3. Análisis de diagnóstico y propuestas para incrementar productividad así como factibilidad (posibilidad de realizarse) y viabilidad (mantenimiento y rentabilidad con recursos disponibles). 4. Generar programa con actividades viables consensuadas y difundirlo; retroalimentando causas de propuestas desechadas. 5. Seguimiento de avances., 6. Comunicar resultados y proporcionar Reconocimiento., 7. Plantear nuevos retos para reiniciar el círculo virtuoso. Para agilizar el proceso se sugiere desarrollar e implementar un sistema basado en tecnologías de la información. Ver Figura 2.

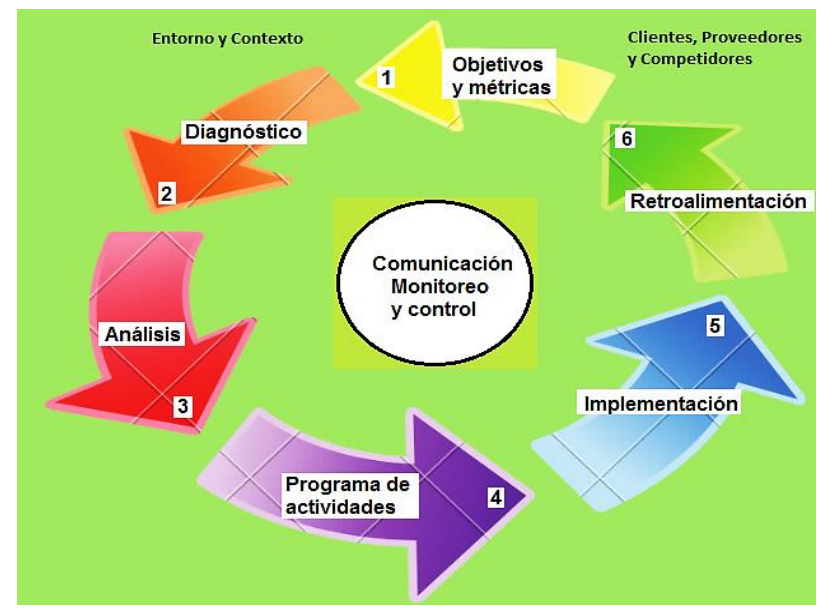

Figura 1 Propuesta inicial de Modelo de Gestión Humana para Incremento de Productividad con enfoque sistémico

Fuente: Laguna- Aguilar, F. (2018)

ISSN-2523-2460

ECORFAN $^{\circledR}$ Todos los derechos reservados

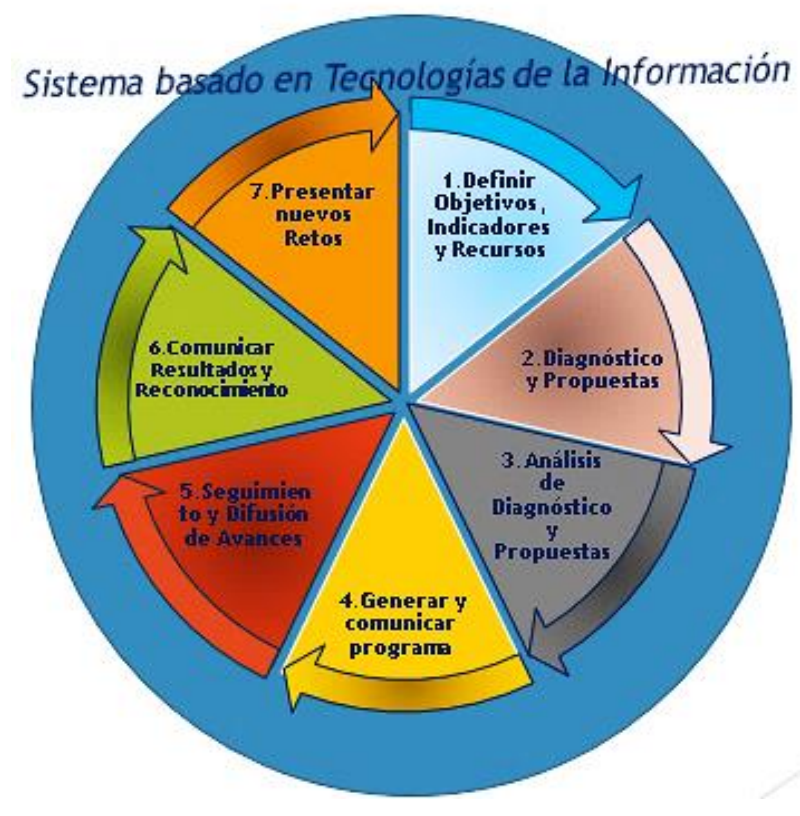

Figura 2 Propuesta inicial de Modelo de Gestión de mejora para Incremento de Productividad con enfoque sistémico

Fuente: Laguna-Aguilar, F. (2019)

En los modelos analizados no todos integran la intervención de las partes interesadas para hacer propuestas hacia el logro de resultados y los reconocimientos con comunicación directa de los recursos disponibles y el uso de tecnologías de la información para esta comunicación, alimentación de información, organización y procesamiento de la misma con seguimiento en la toma de decisiones; alineación de los resultados con los objetivos del personal. Aunque se tocan estos aspectos de forma aislada.

La propuesta busca integrar reconocimientos en función de las necesidades de los participantes de un equipo de mejora, para incrementar la productividad con el trabajo colaborativo, las tecnologías de la información para el monitoreo y control así como los reconocimientos consensuados en función de avances en indicadores y resultados con retroalimentación clara, oportuna, veraz y confiable con la posibilidad de utilizar el internet de las cosas. 


\section{Conclusiones}

Después de este análisis podemos concluir que en la actualidad por la globalización, las organizaciones están inmersas en un mundo competitivo que implica buscar la diferenciación e innovación de sus productos y procesos para mantenerse, crecer y desarrollarse por lo que es necesario llevar a cabo la gestión de la mejora de sus procesos con un enfoque sistémico. La investigación documental de los modelos existentes permitió identificar aspectos coincidentes para la generación de la mejora como es la aplicación del ciclo de mejora aportado por Walter Shewar y difundido por Edward Deming fundamentado en la administración científica de los procesos de Taylor en el siglo pasado, los modelos coinciden en la definición de objetivos, métricas, involucramiento del personal, la comunicación, el reconocimiento del logro y la adaptación de acuerdo a las características muy particulares de cada organización por su cultura organizacional que influye en los modelos de pensamiento, la estructura organizacional, las actitudes y los resultados.

La propuesta integra el determinar desde un inicio los recursos disponibles financieros o materiales para el logro de objetivos así como reconocimiento que se contrastan con los propuestos por su personal, retroalimentando sobre su viabilidad y factibilidad, se programan actividades alineadas a los objetivos e indicadores que a su vez han sido propuestas con la participación del personal, que puede dar seguimiento a sus avances para el logro de los resultados para permitir generar sentimientos de responsabilidad e integración al ser partícipes de las propuestas y ejecución de las mismas con pleno conocimiento de los cursos de acción así como limitaciones por disponibilidad de recursos.

\section{Referencias}

Babé, I. (2002). RE-INGENIERÍA:¿ARREGLO RÁPIDO O SOLUCIÓN A LARGO PLAZO? España: Juran Institute.

Bohoris, G. (1995). A comparative assess ment of some major quality awards. International Journal of Quality \& Reliability Management, 12(9), 30-43. Recuperado el 15 de Febrero de 2019 , de https://doi.org/10.1108/02656719510101178
Crosby, P. (19889). La organización permanece exitosa. México: Mc Graw Hill.

Deming, E. W. (1989). Calidad, productividad y competitividad ala salida de la crisis. Madrid: Díaz de Santos.

EUSKALIT.(2014).

$\mathrm{h}$ ttp://www.euskalit.net/nueva/images/stories/pd fs/modelo20152.pdf. Obtenido de EUSKALIT.net:

http://www.euskalit.net/nueva/images/stories/p dfs/modelo20152.pdf

Fontalvo Herrera, J. T. (2006). LA GESTIÓN AVANZADA DE LA CALIDAD METODOLOGÍAS EFICACES PARA EL DISEÑO,. Santa Fe de Bogotá: ASESORES DEL 2000.

Fundación Iberoamericana para la Gestión de la Calidad. (2015). Fundibeq.org. Obtenido de http://www.fundibeq.org/images/pdf/Modelo_I beroamericano_v2015_FUNDIBEQ-ES.pdf

Ishikawa. (1991). ¿Qué es el Control Total de la Calidad? .La modalidad japonesa. Colobmbia: norma.

Juran, J. (1990). Juran y la planificación de la calidad. Madrid: Díaz de Santos.

Linares Medina, I., \& Ochoa Jiménez, S., \& Ochoa Silva, B. (2013). Cultura organizacional y evaluación del desempeño del personal académico. Estudio de caso en una institución de educación superior pública mexicana. Nova Scientia, 6 (11), 324-345.

López, R. (2001). Obtenido de http://www.jesuitasleon.es/calidad/Modelos $\% 2$ 0de $\% 20$ gestion $\% 20$ de $\% 20$ calidad.pdf

Medina León, D., Noriega Rivera, D., Comas Rodríguez, R., Hernández Nariño, A., Ricardo Cabrera, H., \& Medina Nogueira, D. (2014). La ficha de proceso, soporte del enfoque de procesos y del control de gestión. IX Congresos Internacional de Gestión Empresarial y Administración Pública y el $V$ Taller Internacional de Escuelas y Facultades de Capacitación de Directivos. GESEMAP.

Navarro, E. J. (2017). Fundamentos de la investigación y la innovación educativa. España: UNIR. 